\title{
Interventional Effect of "Group Therapy Based on Problem-Solving Therapy" on the Symptoms of Depression in High School Students
}

\author{
Jin Bin
}

Beijing No.80 high school, Beijing, China

Jinbin201001@126.com

\begin{abstract}
OBJECTIVE: To explore the effect of group therapy based on problem-solving therapy for high school students with depression intervention. METHODS: The grade ten students in Chao yang, Beijing were randomly selected for group therapy intervention based on problem-solving therapy. The intervention last for eight weeks and in every week, the intervention completed by the graduate students of psychology in this city after training for two hours. Use PHQ-9 (nine items questionnaire), ATQ (automated thinking questionnaire) and coping style questionnaire as tools for pre-test and post-test. PREDICTED RESULTS: After the group treatment intervention based on problem-solving therapy has a obvious effect, it can effectively reduce students depressive disorder and negative thinking, and make it more stable to cope with problems. CONCLUSION: Group therapy based on problem-solving therapy has a significant effect on the depression of high school students.
\end{abstract}

Keywords: Group psychotherapy; problem-solving therapy; depression disorder; coping style; high school students.

\section{“基于问题解决疗法的团体治疗” 对高一学生抑郁障碍 症状的干预效果}

靳涁

北京市第八十中学，北京，中国

Jinbin201001@126.com

\section{摘要}

目的：探讨对于高中生抑郁障碍干预的问题解决疗法的团体治疗的干预效果；方法：在北京市朝阳区 高中高一年级随机抽取 100 名高中生进行问题解决疗法的团体治疗干预, 干预共计八周, 每周一次, 一次两个小时, 由本市心理学专业研究生经过培训后来操作完成。运用 PHQ-9 (九条目问卷) 、ATQ（自 动化思维问卷）和应付方式问卷作为前测和后测的工具。预测结果：基于问题解决疗法的团体治疗干 预过后有较为明显的效果, 可以有效减轻学生抑郁障碍, 减少其负性思维, 使其应对问题更稳定。结 论：基于问题解决疗法的团体治疗对高中生抑郁状态及抑郁障碍的干预效果显著。

关键词：团体心理治疗；问题解决疗法；抑郁障碍；应对方式；高中生

\section{1. 前言}

抑郁障碍是一种严重危害人的身心健康的一种疾 病, 并且是一种非常常见的精神障碍。根据世界卫生组 织发布的数据显示, 抑郁症已经成为世界上第四大疾 病, 全球有超过三亿人患有不同程度的抑郁症[1], 而高
中生在这其中占有不小的比例。2005 年至 2013 年大陆 各省多项调查研究显示, 有 $13.1 \% \sim 42.3 \%$ 的中小学生 存在不同水平的抑郁症状 (冯正直, 张大均, 2005; 李小 彩, 赵丽娜, 杨森焙,韩娟, 2010; 朱军红, 钟宝亮, 徐丽 娟, 2013), 并且有相关数据表明抑郁症的复发率是很高 的。处与高中阶段的学生们身心都在快速发展的阶段, 要承受来自身边同学的竞争、父母老师的期望、㚞重课 
业、情感问题等多重心理压力，这些因素提高了他们患 有抑郁障碍的可能性。因此如何提高抑郁障碍治疗在高 中生群体里的可实施性成为了关注点。

抑郁障碍疗法中的团体治疗，也称“小组心理疗法” 是由美国心理学家莫雷诺与 20 世纪初创立。其治疗原 理为利用有一组共同或相似的心理问题的群体, 通过成 员之间的人际互动彼此产生影响, 进而达到治疗的目 的。其治疗方式一般为将受试组成小团体, 由 $1 \sim 2$ 名 治疗者对被试进行治疗, 每次时间约 $2 \mathrm{~h}$ 左右, 小组成 员在此段时间内可以不受拘束地吐露自己内心对真实 想法, 具体治疗技术有行为疗法、精神分析疗法、存在 一人本主义疗法。团体辅导相对于个体辅导具有高效性、 影响时间长的特点, 在像学生群体这样的人群的抑郁障 碍治疗中有着凸显的优势。但是在团体治疗中采用何种 方式更为有效的研究这一方面却存在着空白, 通常采用 多种疗法混合治疗。

问题解决疗法 (PST) , 是一种关注于培养合适的 问题解决态度及技能的认知治疗方法, 其基本理论认为 解决问题需要单独一套认知技能。其目的是改变对问题 的态度, 使其增加积极的问题导向, 减少消极的问题导 向; 改变问题解决的技能, 培养计划型解决问题, 减少 回避型问题解决。其具体技术包括认知和行为这两个方 面, 通过帮助患者识别问题, 将一个大问题划分为若干 个小问题, 针对每一问题写出解决措施及时间, 按照完 成情况在表格上进行标记, 进行进展回顾, 当患者发现 自己解决问题能力提高时, 便会改变对自己对消极态 度。在操作方面治疗周期较短。不需要高水准的心理咨 询师进行操作, 而是具备一定心理学基础的并经过简单 培训人就可以操作的一个疗法。问题解决虽有很多优 势, 但由于此疗法提出时间较晚, 起源于国外导致国内 在此疗法领域内存在空白, 尤其是高中生治疗效果方面 较少, 也并没有得到广泛应用。

在此基础上, 本实验针对基于问题解决疗法的团体 治疗及基于抑郁障碍心理教育的团体治疗对于高中生 的抑郁障碍干预效果进行研究。实验将在本市选取 100 名高一年级不同性别的学生, 在干预开始前做相同的三 个量表—九条目问卷(PHQ-9)自动化思维问卷(ATQ) 及应付方式问卷, 统计数据作为前测结果。在接下来为 期两个月的过程中, 100 名学生被随机分为 10 小组分别 进行基于问题解决疗法的团体治疗, 分别在第四周和第 八周的治疗过后进行九条目问卷、应付方式问卷及自动 化思维问卷的填写, 统计数据。预测该两组实验前与实 验后的量表差值有较大的变化, 基于问题解决疗法的团 体治疗对于高中学生干预效果明显。

\section{2. 研究方法}

\section{1. 被试}

研究在北京市朝阳区的高中一年级选取不同性别 的学生 100 人, 统计其性别等基本情况。在征得同意后
并保证正常学习生活不被影响的情况下，向他们说明本 次试验的目的及要求, 知情同意者发放九条目问卷、应 付方式问卷及自动化思维问卷。

\section{2. 研究材料}

对于学生心理状态的测量, 本次试验使用的是九条 目问卷、应付方式问卷及自动化思维问卷。PHQ-9 问卷, 它不是抑郁症的篮查工具, 但可用于监测抑郁症的严重 程度和对治疗的反应。共有 9 个条目即 9 个抑郁症状组 成即九个抑郁症状组成, 分别是 1 、愉快感丧失, 2 、心 情低落，3、睡眠障碍，4、精力缺乏，5、饮食障碍，6、 自我评价低，7、集中注意力困难，8、烦燥不安，9、

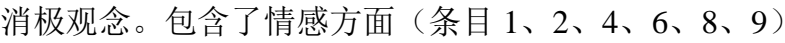
及躯体症状方面（条目 3、5、7）两个维度，九条目问 卷内容简介, 填写简便, 关注的是参与者自身两周内的 情况, 分数越高则抑郁障碍越严重。有关实验表明九条 目问卷有较高的信效度、敏感性和特异性。评分标准为 0-4 分没有抑郁, 5-9 分有轻微抑有, 10-14 分有明显抑 郁症状，15-27 分属于重度抑郁。

ATQ 即自动化思维问卷, 是 Hollon\&Kendall 于 1980 年研究设计而成, 该问卷通过让受试回忆自己最近一周 30 种不同想法出现的频度，频度分为五个等级：1=无； $2=$ 偶尔; $3=$ 有时; $4=$ 经常; $5=$ =持续存在, 问卷切实反映 出对自我认知对评价。问卷涉及抑郁的四个方面: 1 .个 体适应不良及对改变的苛求; 2 .消极的自我概念与消极 的期望；3.自信不足； 4, 无助感 [2]。可以有效的区分 抑郁和非抑郁的受试, 总分为 30 (无抑郁或轻度抑郁) 到 150 (极度抑郁)。ATQ 内部一致性很高, 信效度测 试结果良好。

应付方式问卷，是由肖计划等人参考: Billings\&moos(1980),Folkman\&Gardner(1980),Ilefld(198 0)Ray\&Lindop(1982),Bond\&Gardner(1983)以及 Stone 和 Neale(1986)等人研究应付和防御时所用的问卷内容以 及有关“应付”的理论思想, 根据汉语特点进行设计的。 分为解决问题即求助成熟型, 此类人常能解决问题或求 助他人; 退避即自责不成熟型, 此类人通常以退避、幻 想来面对困难和挫折; 合理化混合型, 此类人集成熟和 不成熟与一身, 具有双面人格特点。对于进行基于问题 解决疗法的团体治疗引导员, 均为本市心理学专业的研 究生,在试验前接受 $12 \mathrm{~h}$ 的培训。

\section{3. 实验过程}

被试在同一周开始接受干预，干预每周六进行一 次, 每次时长为两小时。活动场所为同一个固定的教室, 环境较舒适。十个小组由同样的接受过培训的心理学专 业的十个研究生进行干预, 同样进行基于问题解决疗法 的团体治疗干预。每小组分别由一位心理专业的研究生 进行团体治疗引导。在干预开始前进行自动化思维问 卷、九条目问卷及应付方式问卷的填写, 作为前测结果。 
基于问题解决疗法的团体治疗步骤：问题解决疗法 分为七个阶段:

第一到二周：被试互相了解对方，两人一组接受问 题解决疗法干预, 每两人配备 1 名研究生。研究生先阐 明整个疗程的步骤以及进行治疗的规章制度。引导其明 确面对问题的态度, 被试学习如何以积极的态度面对问 题，帮助受试拒绝生活中的问题是个人缺陷的信念。帮 助受试识别问题情境的外部原因而不是假设的自我内 部和负面归因。

具体方法为: 1 . 权衡证据 查找患者没有问题的生活 区域的问题列表表单。2.角色扮演 尝试改变患者的角 色，让患者戴上不同的帽子，成为客观倾听者或朋友， 而不是因负面情绪状态而存在偏见的抑郁个体。3.停下 来思考 可以帮助被试更有规划地考虑自己要干什么。

第三周: 引导其列出自己的问题清单。具体方法为: 首先通过患者自由报告列出容易辨认、最明确的问题。 其次通过线索找到不易发现的问题。最后由研究生辅助 其运用问题检查清单寻找自己潜在领域的问题。

表一 问题检查清单

\begin{tabular}{|c|c|l|}
\hline 1 & $\begin{array}{c}\text { 和同学、老师、朋友的 } \\
\text { 关系 }\end{array}$ & \\
\hline 2 & 和家人的关系 & \\
\hline 3 & 失恋 & \\
\hline 4 & 亲人朋友离世 & \\
\hline 5 & 被隔离、孤独 & \\
\hline 6 & 学习或工作问题 & \\
\hline 7 & 金钱问题 & \\
\hline 8 & 法律问题 & \\
\hline 9 & 住房问题 & \\
\hline 10 & 伤害、勒索、虐待 & \\
\hline 11 & 不良嗜好问题 & \\
\hline 12 & 身体健康 & \\
\hline 13 & 性问题 & \\
\hline 14 & 心理健康问题 & \\
\hline 15 & 低自尊或不自信 & \\
\hline
\end{tabular}

第四周: 选择和定义问题。由被试从问题清单选择 一个问题, 通过研究生的辅助将大问题化解为若干个小 问题, 并清晰客观地陈述问题和目标。研究生引导其进 行头脑风暴生成解决方案

第五周：做决定并形成和实施 SMART 并执行计划 (即 $S$ 明确化、 $M$ 可量化、 $A$ 可实现的、 $R$ 与行为、效
能相关的、 $\mathrm{T}$ 有时间规定的）计划。具体方案为: 对上 一周所罗列的每项解决方案, 罗列支持和反对的观点, 评估并选择解决方案, 将方案细分成几个小且可执行性 强的步骤, 并给每一个步骤都制定一个完成的时间节 点, 执行计划。

第六周：对行动计划进行检测，目的在于监察计划 的实施情况，调整在实施中出现的问题并解决在执行过 程中所遇到的困难，完善 SMART 计划，在下一周再次 执行计划。

$$
\text { 表二 进展回顾表 }
$$

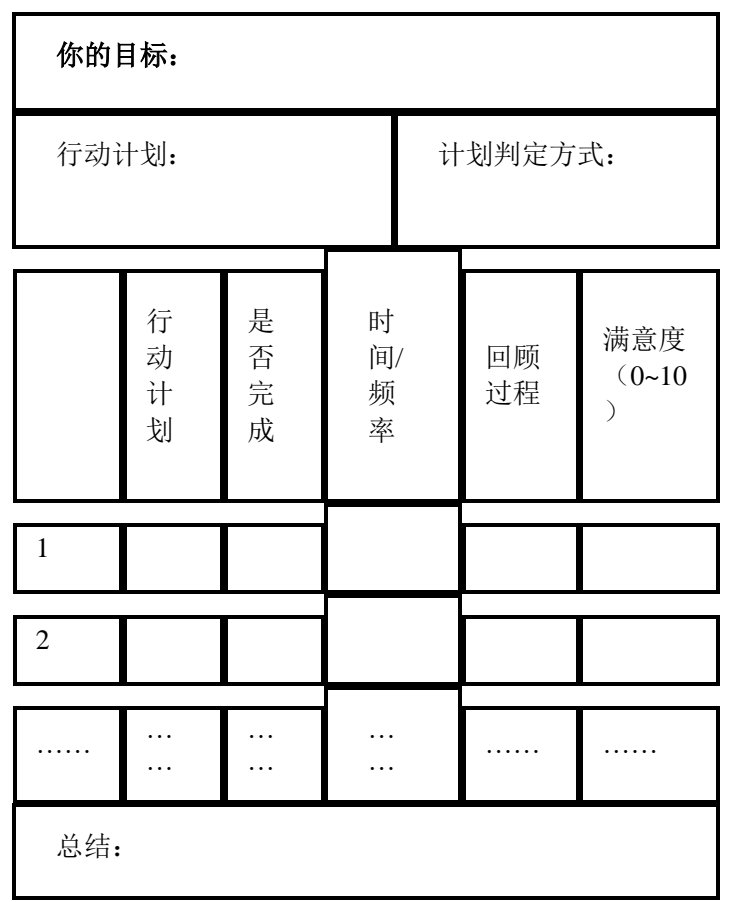

第七周: 再次进行进展回顾, 可以适当添加 SMART 计划中的内容并且在接下来一周继续执行新计划。

第八周：养成习惯，回顾整个问题解决疗法在被试 身上的使用方法及整个干预过程中的收获，总结自身执 行中的优缺点, 谈谈在干预过程中的感受

在干预结束之后被试进行自动化思维问卷、九条目 问卷和应付方式问卷的填写, 其数据作为后测的结果。

\section{4. 数据的收集与分析}

第一次团体治疗之前、最后一次团体辅导后以及干 预两个月后分别用九条目问卷、自动化思维问卷及应付 方式问卷来评估学生抑郁成都市使用 SPSS17.0 和 HLM6.08 软件进行数据方差分析

\section{3. 预测结果}

预测两个月后，九条目问卷显示有抑郁的被试干预 后九条目问卷分数有明显的下降，自动化思维问卷分数 
较高的被试在干预后分数有多下降, 负性思维减 少。此次干预会将其问题应对变得更稳定。

\section{4. 讨论}

在干预效果上, 本研究与近年来的研究的结论一 致, 也与本研究的预期结果一致。基于问题解决疗法的 团体治疗干预显著降低了该被试组的量表分数。

研究证明了基于问题解决疗法的团体治疗对于缓 解高一学生抑郁障碍有所效果, 可以有效减少高一学生 的负性思维, 使其问题应对变得更稳定, 并且问题解决 疗法对于高中生抑郁状态及障碍的针对性强、治疗效果 明显。但是, 仍存在着一些局限。首先由于条件限制, 本次的干预方案只涉及到一个区的高一年级部分高中 生, 取样缺乏代表性。但本次抽样的学校均为公立高中, 并且样本量较为充足, 取样方式为随机取样, 具有较强 的科学性。因此, 本次干预方案还是具有一定的代表性。 其次, 本次干预持续时间较短, 测试结果可能具有偶然 性。

本研究的方法及结果可以广泛应用于高中生抑郁 状态及抑郁障碍的干预当中, 可以较为有效的帮助高中 生缓解其抑郁状态及抑郁障碍, 对症下药, 促进其心理 健康, 使其能够妥善处理学习生活中遇到的问题, 拥有 心理健康的人生, 并带动身边的人一起积极面对抑有状 态及抑郁障碍。

\section{5. 结论}

问题解决疗法在针对高中生抑郁障碍及抑郁症干 预上有显著的成效, 可以有效降低高中生负性思维, 使 其问题应对变得更加稳定, 可以推广到高中生乃至整个 学生群体中。

\section{References}

[1] FengZ, Zhang Dajun. (2005). Epidemiological characteristics of depression symptoms in middle school students. Chinese Journal of Behavioral Medical Sciences, 14 (2), 103-105.

[2] World health organization (2017). Depression and Other Common Mental Disorders

[3] Lin Z. (2003) Psychology Dictionary (Volume 2). Shanghai Education Press.

[4] Wang X, Ren X. (1999) Mental Health Assessment Scale Manual (Revised Edition). China Mental Health Magazine, Beijing.

[5] Xu Y,Wu H,Xu I.(2007)Application of the Patient Health Questionnaire Depression Scale (PHQ-9) in the elderly in the community-Reliability and validity analysis. Shanghai Psychiatry.

[6] Song L, Yu X, Zhang H. (2013). The effect of group psychotherapy on the efficacy and quality of life of patients with depression, Chinese Journal of Health Psychology., Vol21. No 1

[7] Sun L, Feng W. (2008) New progress in research on adolescent depression intervention, Modern Preventive Medicine, Vol35. No.20

[8]de Man-van Ginkel JM, Gooskens F, Schepers VP, et al. (2012). Screening for poststroke depression using the patient health questionnaire. Nurs Res. 20. Sep-Oct 61(5):333-41.

[9] Si S, Lin X, Liu J (2015) The effect of positive psychological group counseling on depression symptoms of primary and middle school students. Psychological Science, 38 (4): 1012-1018

[10] Arthur M. Nezu; Christine M. Nezu; Holly R. Gerber. (2019) (Emotion-centered) problem-solving therapy: An update. Australian Psychologist. 